\title{
A Macintosh analogue of the rotary pursuit task
}

\author{
DANIEL B. WILLINGHAM, JEFF HOLLIER, and JANE JOSEPH \\ University of Virginia, Charlottesville, Virginia
}

\begin{abstract}
Pursuit tracking, a perceptual motor-skill task, has had wide application in many areas of psychology. The task is usually administered with a mechanical device in which subjects try to maintain a contact between a stylus and a disk on a rotating platter. A pursuit-tracking task that can be administered on a Macintosh computer is described. Subjects try to keep a crosshair cursor on top of a moving circular target. The cursor can be controlled by commonly used Macintosh ADB devices such as a joystick. The computer version has several important advantages: it offers more task flexibility and more accurate dependent measures of performance, and it is inexpensive.
\end{abstract}

Pursuit tracking, in which a subject uses a hand-held device, such as a stylus, to track a moving target, has been used in many areas of psychology, including human learning (Ammons, 1947; Koonce, Chambliss, \& Irion, 1964), social psychology (Jourden, Bandura, \& Banfield, 1991), personality (Randall, Crabtree, \& Antrim, 1989), psychopathology (Thirer, Zackheim, \& Summers, 1987), and neuropsychology (Eslinger \& Damasio, 1986; Heindel, Salmon, Shults, Walicke, \& Butters, 1989).

Most of these pursuit-tracking studies use the standard mechanical rotary-pursuit device (e.g., Lafayette Instruments, Lafayette, IN, No. 30010), which consists of a platter (approximately $25.5 \mathrm{~cm}$ in diameter) that rotates. There are four speed selections: $15,30,45$, and 60 rotations per minute (rpm). On the platter is a small disk (approximately $1.9 \mathrm{~cm}$ in diameter). Newer versions use optical tracking and offer three patterns of target movement-square, circle, and triangle (e.g., Lafayette Instruments, No. 30020) - and a more advanced model includes flexible speed setting from 0 to $100 \mathrm{rpm}$ (e.g., Lafayette Instruments, No. 30014). The subject holds a stylus in his or her preferred hand and tries to keep the tip of the stylus in contact with the small disk as the platter turns (or in contact with the light as it moves, in the case of the optical tracking models). The dependent measures for these mechanical devices are the amount of time during the trial that the stylus is on the target, the number of times the stylus loses contact with the target, and the average time it takes the subject to regain contact with the target.

\section{Advantages of a Computer Version of Pursuit Tracking}

Implementing an analogue of the rotary-pursuit task on a personal computer offers several important advantages. First, the dependent measure is more precise. Our

Correspondence should be addressed to D. B. Willingham, Department of Psychology, University of Virginia, Charlottesville, VA 22903-2477 (e-mail: dbw8n@virginia.edu). program samples the position of the target and cursor every $200 \mathrm{msec}$, and so one can calculate the distance between the cursor and the center of the target across the trial, rather than simply measuring whether the stylus is on the target or not. This advantage is particularly important given that apparent level of learning and even the shape of the learning curve can vary depending on the size of the target used in a tracking task, which naturally leads to different measures of performance, if time on target is the dependent measure (Bahrick, Fitts, \& Briggs, 1957). Time on target can of course be derived from the distance information as well. Second, the program records to a file on disk all of the coordinates of the target and cursor sampled during a trial, so that other analyses can be conducted later. This information can be quite useful if, for example, a subject group tends to track quite consistently but to lag $3 \mathrm{~cm}$ behind the target. A simple time-on-target measure would correctly show that subjects were not on target during the trial, but would not show that subjects were tracking the target well. A third advantage is that the computer version of the task makes it easy to change the pattern in which the target movesit need not be a circle. Indeed, the target need not move in a predictable pattern at all. A fourth advantage is cost. Our computer version uses equipment present in most laboratories, whereas the rotary-pursuit devices described above (with timing devices) cost between $\$ 715$ and $\$ 2,040$.

Other researchers have used a joystick and computer screen or oscilloscope to test pursuit tracking (e.g., Pew, 1974; Poulton, 1974). The programs controlling these tasks have not been made widely available, however. To our knowledge, a Macintosh pursuit-tracking program is not readily available.

\section{A Macintosh Analogue of Rotary Pursuit}

Our task is administered on the Macintosh computer, and is written in THINK Pascal (Siegel, McEnerny, \& Neal, 1991). The subject can track the target using any device that manipulates the cursor--a mouse, trackball, digitizing tablet, touchscreen, and so on - although there 
are some factors to consider in choosing a device, which are described below. We have used a joystick (Advanced Gravis optical joystick, Advanced Gravis Computer Technology, Burnaby, Canada). The program runs properly on all Macintoshes that use the 68030 processor chip and run at a clock speed of $33 \mathrm{MHz}$ (e.g., Mac IIci), and all Quidras and Power Macintoshes. Although the program runs on slower Macs, there is an upper limit on target speed because of the slower processor, and we recommend that this program not be used on those computers. A menu command allows the user to change the size of the target path so that the program can be used with PowerBook computers.

The subject sees a 1.06-cm-diam dot (hereafter called the target) and positions a cross-hair cursor on top of the target. The subject initiates a trial by clicking the mouse button. The target moves on the screen, and the subject attempts to move the cursor so that it is superimposed on the target.

The experimenter controls (1) the target path (square, diamond, octagon, or random), (2) direction of target movement (clockwise or counterclockwise) if the path is not random, (3) target speed, and (4) number of trial blocks to be presented and number of trials in each block. The program also offers standardization trials, which allow the experimenter to change the speed of the target after each trial in order to obtain subject performance near some desired standard. Standardization trials are explained in greater detail below.

Target movement. The movement of the target is simulated by continually erasing the target and redrawing it in a new position. The apparent speed of the target depends on two factors: the delay between erasing the old target and drawing the new target and the distance between the erased and redrawn targets. Varying the distance to vary the speed is limited by the 2-D coordinate system of the Macintosh screen. For example, suppose one chose to move the target five pixels between each screen refresh. That value must remain constant if the speed is to remain constant. That is easy enough if the target moves only northward $\left(0^{\circ}\right)$, southward $\left(180^{\circ}\right)$, eastward $\left(90^{\circ}\right)$, or westward $\left(270^{\circ}\right)$. Other directions create problems, however, as illustrated in Figure 1.

Figure la shows what happens if one tries to move southeastward $\left(135^{\circ}\right)$. If one travels five pixels in that direction, one will travel farther than when moving eastward. One can solve the problem (in this case) by moving three pixels eastward and four southward, but that solution means that the target is not moving southeastward at $135^{\circ}$, as shown in Figure $1 \mathrm{~b}$. Thus, although the apparent speed of the target can be changed by changing the distance between the erased and redrawn target, this strategy is severely limited by the coordinate system of the screen.

The present implementation addresses this problem and allows for the drawing of horizontal, vertical, and diagonal movement. The distance between the erased and the redrawn targets does not vary; instead, when the target moves horizontally or vertically, the new target is drawn 7 pixels (approximately $2.47 \mathrm{~mm}$ ) from the erased target. When the target moves diagonally, the target position is changed 5 pixels horizontally and 5 pixels vertically, which yields a diagonal distance of approximately $2.49 \mathrm{~mm}$. This solution is not perfect, but the diagonal distance is obviously very close to the desired value.

Target path. The target movement can be either patterned or random. The program currently supports three patterns: a diamond, a square, and an octagon (to approximate the circular pattern used in the mechanical versions of the rotary pursuit task). The user defines whether the target should move in a clockwise or counterclockwise direction.

On patterned trials, the boundary of the pattern is visible on the screen. We have implemented the program this way to match the mechanical version of rotary pursuit, in which it is apparent to the subject that the target will follow a circular path because the platter is round. The screen is black except for a central white shape (square, diamond, or octagon) that represents the target path. The boundary that appears in our version can be re-

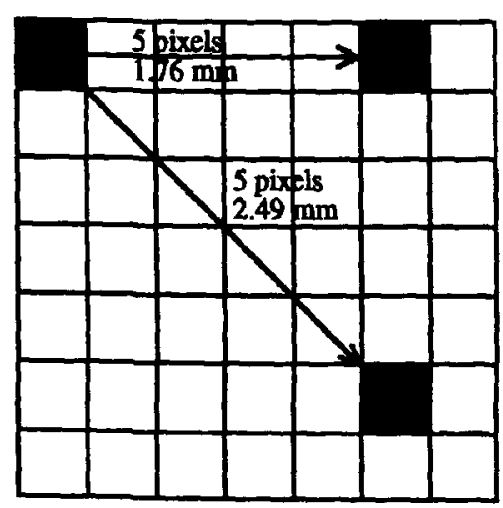

a

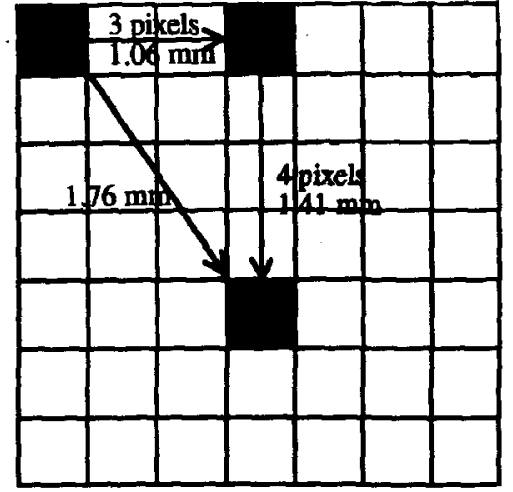

b

Figure 1. Ilustration of the change in distance that results from a change in direction on a two-dimensional coordinate screen. 
moved by changing the source code so that the entire screen is white. The target path is slightly bigger than the screen of a Macintosh Classic but the size of patterns can be changed via a single command in the source code.

There is not a special limitation on patterns (we have also pilot-tested a star). The only limitation on patterns at present is that the pattern be composed of horizontal, vertical, and diagonal lines.

For random movements, the target initially moves in one of eight randomly selected directions (north, south, east, west, northeast, southeast, southwest, northwest). Each time the target is to be redrawn, there is a probability of the target's moving in a new, random direction. The probability is set by the user, and the program tells the user which settings lead to an average number of direction changes equal to the square, diamond, or octagon.

Target speed. Varying distance to adjust the speed is not practical (as discussed above), so we vary speed by varying the delay between erasing the target and redrawing it. If the target is erased and redrawn on successive screen refreshes, the speed of the target is quite rapid and difficult to track. Therefore, the program draws the target, waits during a specified delay, then erases the target and immediately redraws it.

The drawing of the target is necessarily synchronized with the screen refresh rate so that delays must occur in increments of $16.7 \mathrm{msec}$ (usually referred to as 1 tick). However, to allow finer resolution of apparent target speed, increments of $1 / 2$ tick are implemented as follows. If a delay of 2.5 ticks is specified, the delay is actually 2 ticks before the next redrawing, 3 ticks before the following redrawing, 2 ticks before the following redrawing, and so on. This allows for apparent target speed to be on average between 2 and 3 ticks, or $59.3 \mathrm{~mm} / \mathrm{sec}$.

Given that the target moves 7 pixels and that as much as $50-100 \mathrm{msec}$ might pass between successive drawings, it might seem that target movement would not appear smooth. The phi phenomenon is powerful, however, and the subjective experience of subjects we have tested is of a relatively smoothly moving dot.

Because performance levels among subjects may vary widely (particularly if neurological patient populations are tested), the program supports the capability to vary the target speed in order to standardize each subject's performance so that all subjects start at the same level of performance before the training trials. This capability is described below.

Trial types. The program supports two types of trials. Standardization trials are brief $(15 \mathrm{sec})$ and provide immediate feedback about performance (root mean squared error, standard deviation of distance between target and cursor, and percent time on target). Root mean square error is a measure of distance between the target and the cursor. Error on each trial is calculated by forming a right triangle-one leg is vertical distance between target and cursor, and the other leg is horizontal distance. The hypotenuse is the distance between the cursor and target, or error. Those errors are squared and summed.
Then the square root of the mean of the summed errors is taken.

The feedback is based on the last $10 \mathrm{sec}$ of the trial because performance in the first $5 \mathrm{sec}$ tends to be quite variable. The experimenter has the opportunity to change the delay (and thereby the speed) after each trial. Thus, the experimenter can adjust the target speed so that each subject begins the experiment at approximately the same level of performance, a strategy that has been employed in the neuropsychological literature (Heindel et al., 1989; Singh et al., 1992). The program supports standardization trials for both patterned and random movement. We have found that three to five trials is sufficient to obtain performance in the desired range. Some learning probably occurs on the standardization trials, so, to minimize the subject's opportunity to gain skill, we have made these trials brief.

Training trials last $25 \mathrm{sec}$. The speed used for training trials can be based on the subject's performance on the standardization trials. The program provides feedback after each trial (root mean squared error in pixels, standard deviation of root squared errors, and percentage of trial time on target). The user defines how many trials are to appear in a block and how many blocks are to be administered. A $60-\mathrm{sec}$ rest break occurs between trial blocks.

Data collection. The program records the location of the cursor and the center of the target every $200 \mathrm{msec}$. These raw data are recorded to a file on disk for both standardization and training trials. A summary of the data from each trial is also recorded in a separate file. The summary includes the mean distance, measured as root mean squared error, and the standard deviation of the raw distance for each trial. The first $5 \mathrm{sec}$ of each trial are not included, because that data tends to be quite variable; although the subject initiates movement of the target, it takes a few seconds for him/her to "catch up" to the target.

\section{A DEMONSTRATION EXPERIMENT}

We performed a minimal test of the task to be sure that subjects learn to track as expected.

\section{Method}

Subjects. Twenty-four undergraduates ( $12 \mathrm{men}, 12$ women) at the University of Virginia participated to fulfill a course requirement.

Design. The subjects performed five blocks of trials with the target moving randomly and then five blocks with the target moving in a repeating pattern. There were four trials in each block and a 45 -sec rest period between blocks. During the pattern phase of the experiment, half of the subjects saw the target move counterclockwise in a square pattern and half saw it move clockwise in a diamond pattern. The speed of the target was individually set for each subject, dictated by performance on several standardization trials before the random task and before the pattern task. The experimenter set the speed during standardization trials so that root mean squared error was $30-35$ pixels. 
Procedure. Testing was conducted in a quiet, moderately lit room. Spring tension on the joystick was released so that the joystick moved freely. The task was administered as described earlier.

\section{Results}

The primary dependent measure of interest is root mean squared error. The median was calculated for each block of trials. The means of those medians are shown in Figure 2.

The medians were analyzed in an analysis of variance. For the random target movement, subjects' performance improved reliably across trial blocks $\left(F=18.67, M S_{\mathrm{e}}=\right.$ $5.94, p<.001$ ). There was not a reliable effect of condition (square vs. diamond; $F=0.001, M S_{\mathrm{e}}=94.97, p>$ $.20)$ and no interaction $\left(F=0.57, M S_{\mathrm{e}}=5.94, p>.20\right)$. Indeed, one could not expect an effect, inasmuch as this was the random pattern preceding the repeating pattern.

Subjects also learned the repeating pattern, as shown by a reliable effect of trial block $\left(F=15.59, M S_{\mathrm{e}}=\right.$ $4.22, p<.001$ ). There was not a reliable effect of condition (square vs. diamond; $F=0.66, M S_{\mathrm{e}}=51.26, p>$ $.20)$ and no interaction $\left(F=1.78, M S_{\mathrm{e}}=4.22, p=.14\right)$. Thus, subjects learned the diamond and the square, and learned each at the same rate.

The range of standardization trials performed was quite small-between 2 and 5 . The speed did differ between the random and pattern trials $\left(F=15.75, M S_{\mathrm{e}}=0.07\right.$, $p<.001$ ); the average speeds were 2.79 ticks for the random trials and 1.65 ticks for the pattern trials. There was no difference in speed for the square and diamond conditions $\left(F=0.66, M S_{\mathrm{e}}=0.14, p>.20\right)$.

Despite the differences in speed, the subjects did not start the random and pattern tasks at equal performance levels. The goal was to start performance with an error

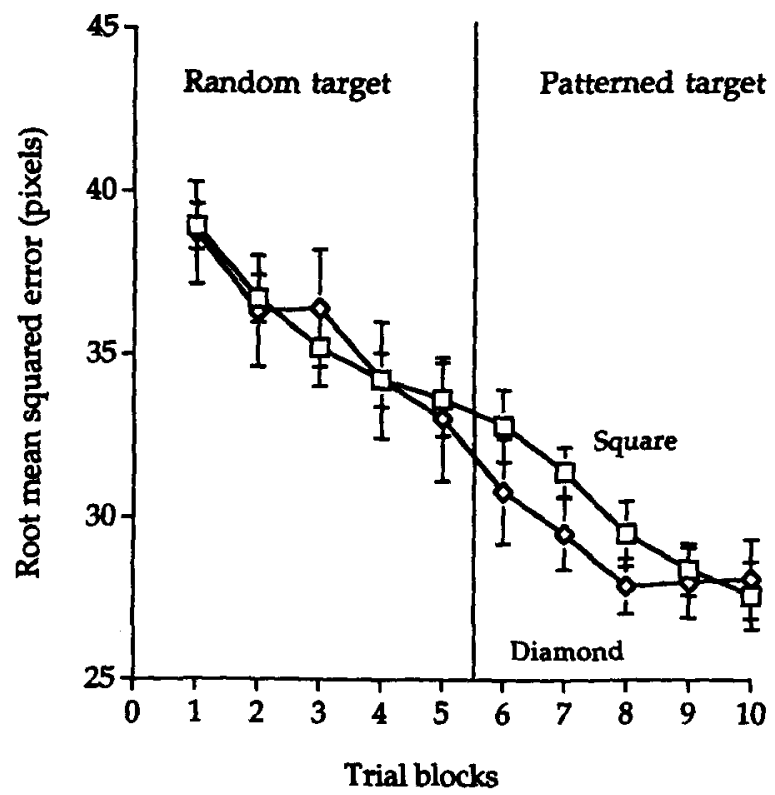

Figure 2. Root mean squared error across trial blocks when the target moves randomly and in a repeating pattern. Error bars are standard errors of the mean. of approximately 35 pixels. However, the actual initial error was slightly higher than this goal on the random task and slightly lower on the pattern task.

In summary, the important point is that subjects showed robust learning when the target moved randomly or in a pattern following a diamond or a square, thus demonstrating the viability of our computerized version of pursuit tracking.

\section{DISCUSSION}

\section{Limitations}

Certain changes to the program are quite easy to implement, including changing the target pattern (so long as horizontal, vertical, and diagonal segments make up the pattern), the size of the target, or the mapping of input device to cursor movement (e.g., implementing a mirror reversal). Some of these changes require a single line of code, some more, but all are conceptually simple to instantiate. Other changes are not so simple to make.

Curved patterns are more troublesome to generate than linear patterns, because the direction of the target must be generated by a function. Also, the pattern will always be an approximation, again due to the two-dimensional coordinate system on which the Macintosh screen is based. A bigger problem is that the distance the target moves each time it is erased and redrawn may not be constant (because the path is an approximation), so it would be necessary to recalculate the delay for each redrawing. In principle, nothing prevents the use of curved patterns, but the program does not lend itself to simple modification for the use of curved patterns.

A more troubling limitation is the use of the system clock for timing. The Macintosh system clock marks time in 16.67-msec "ticks." An ADB input device (such as a joystick) will be polled irregularly-at some time between every 8 and $16 \mathrm{msec}$. So, although the program attempts to record the coordinates of the target and the cursor every $200 \mathrm{msec}$, it is recording every $200 \mathrm{msec}$ on the average; the time window within which the coordinates are sampled may be as large as $32 \mathrm{msec}$. Although this resolution is not desirable, it should be borne in mind that the dependent measure is distance, not response time. The program samples distances at intervals that are somewhat irregular, but this should not lead to deceptive results.

This timing limitation also means that, as with all programs where timing is important, the user must be certain that there are not other functions (e.g., inits, control panel devices, network activity) stealing CPU time from the rotary pursuit program. The easiest way to do this is to place the rotary-pursuit program on the computer's hard drive and to boot the machine from a floppy diskette that contains a minimal system version of System $6.0 \mathrm{x}$. A minimal system is one without inits or control-panel devices other than those necessary to drive whatever peripheral device (e.g., a joystick) is being used to collect data. If System 7.x is being used, booting the computer with the shift key depressed will disable extensions. 
Timing is also an issue for the scaling of the mouse and other ADB devices. The ADB is polled for mouse, and therefore cursor, location approximately once every screen refresh. The Macintosh operating system maintains an acceleration scheme for relative pointing devices, that is, mice and mouse replacements such as trackballs. Such a scheme allows for slow mouse movements, fewer than 6 pixels per refresh, in order to map to cursor movement in a one-to-one fashion. However, mouse movements greater than 6 pixels per refresh move the cursor twice as far horizontally and vertically (Apple Computer, 1985, p. 372). In this task, target movement is slower than 6 pixels per screen refresh, but a subject correcting a large tracking error could easily exceed this threshold. The current version of the Cursor Device Manager allows for better control over relative pointing devices (Apple Computer, 1994). It is important that the pointing device employed during testing with our task utilize a custom acceleration strategy, that is, uses a custom "accl" resource. The experimenter must be able to shut off any acceleration schemes employed either by the operating system or by the ADB driver software controlling the pointing device.

A final warning concerns the extent to which results from this task are readily comparable to results from published tracking studies. It is possible that tracking a pattern composed of straight lines (as in this task) yields performance that is different from that used for tracking a circle (as in rotary pursuit). It is also possible that performance on our task depends, in part, on the pointing device used (joystick, trackball, etc.). It is not practical to test all input devices for comparability to rotary pursuit under all of the conditions for which rotary pursuit has been used, so researchers should not assume that this task is interchangeable with the mechanical rotarypursuit task.

\section{Summary}

The Macintosh pursuit-tracking task that we have developed offers significant advantages over the mechanical rotary-pursuit task: cost, task flexibility, accuracy of measurement, and flexibility of dependent measure. There are limitations, as noted above, but the advantages over mechanical versions of rotary pursuit are salient. Still, comparability to the mechanical version is not guar- anteed, so if comparability is important, the mechanical version is the better choice.

\section{Availability}

The application program is available by anonymous ftp from the Macpsych archive. Source code is available directly from the authors.

\section{REFERENCES}

Ammons, R. B. (1947). Acquisition of motor skill: II. Rotary pursuit performance with continuous practice before and after a single rest. Journal of Experimental Psychology, 40, 393-411.

APPLE Computer (1985). Inside Macintosh, Vol. II. New York: Addison Wesley.

APPLE COMPUTER (1994). ADB-The untold story: Space aliens ate my mouse. Macintosh Developer Support New Technical Notes. Cupertino, CA: Author.

Bahrick, H. P., Fitts, P. M., \& Briggs, G. E. (1957). Learning curves - facts or artifacts. Psychological Bulletin, 54, 256-258.

Eslinger, P. J., \& Damasio, A. R. (1986). Preserved motor learning in Alzheimer's disease: Implications for anatomy and behavior. Journal of Neuroscience, 6, 3006-3009.

Heindel, W. C., Salmon, D. P., Shults, C. W., Walicke, P. A., \& ButTERS, N. (1989). Neuropsychological evidence for multiple implicit memory systems: A comparison of Alzheimer's, Huntington's, and Parkinson's disease patients. Journal of Neuroscience, 9, 582-587.

Jourden, F. J., Bandura, A., \& Banfield, J. T. (1991). The impact of conceptions of ability on self-regulatory factors and motor skill acquisition. Journal of Sport \& Exercise Psychology, 13, 213-226.

KoOnce, J. M., Chambliss, D. J., \& Irion, A. L. (1964). Long-term reminiscence in the pursuit-rotor habit. Journal of Experimental Psychology, 67, 498-500.

PEw, R. W. (1974). Levels of analysis in motor control. Brain Research, 71, 393-400.

Poulton, E. C. (1974). Tracking skill and manual control. New York: Academic Press.

Randall, T., Crabtree, D. A., \& Antrim, L. (1989). Relations between personality measures and physiological reactivity using a perceptual motor challenge. Perceptual \& Motor Skills, 69, 944-946.

Siegel, R., McEnerny, J., \& Neal, D. (1991). THINK Pascal. Cupertino, CA: Symantec Corp.

Singh, J., Gabrieli, J. D. E., Willingham, D. B., Kirschner, K., Stebbins, G. T., \& GoetZ, C. G. (1992). Dissociable components of sensory-motor skill learning in patients with Huntington's disease. Society for Neuroscience Abstracts, 18, 1212.

ThIRER, J., ZACKHEIM, M. A., \& SUMmers, D. A. (1987). The influence of depression on selected motor performance tasks by college athletes and nonathletes. Educational \& Psychological Research, 7, 75-89.

(Manuscript received July 6, 1994: revision accepted for publication October 6, 1994.) 